\title{
Material in Maxillofacial Prosthodontics - A Review
}

\author{
Surekha Godbole Dubey ${ }^{1}$, Tanvi Rajesh Balwani², Aditi Vinay Chandak³ ${ }^{3}$ Samidha Pande ${ }^{4}$ \\ 1,2,3,4 Department of Prosthodontics, Sharad Pawar Dental College, Sawangi, Maharashtra, India.
}

\section{ABSTRACT}

As there is an increase in the rate of oral cancer, the surge for the various maxillofacial materials is increasing as well. Materials used for rehabilitation of congenital or acquired defects of patients should fulfil the requirements such as function, longevity, and aesthetics. Defects of face which may be congenital or acquired makes the condition of the affected person very disastrous mentally, physically as well as emotionally. Patient wants facial rehabilitation for a healthy happy life. To provide rehabilitation of these patients, re-evaluation of materials used in the field of maxillofacial prosthesis seems essential. Maxillofacial material should best suit the ideal selection criteria to satisfy the functionality, biocompatibility, aesthetics as well as durability. While the new materials have exhibited many desirable properties, there are also many insufficiencies involved in it. This article presents a systemic review of the evolution, current trends, and future requirements in maxillofacial materials for ensuring psychological well-being.

In the recent years, a dramatic increase in the demand for prosthetic rehabilitation of patients with facial defects is seen. Increasing awareness of cancer is resulting in earlier diagnosis and treatment. Unfortunately, many of the surgical techniques are extensive and thus leave large defects that compromise not only function and aesthetics, but also psychosocial status of the patient. These problems require prompt rehabilitation with surgery or prosthetics. ${ }^{1}$ However, surgical reconstruction is often contraindicated in the presence of large defects or in high-risk patients. Prosthesis offers the advantage of quick, reversible and medically uncomplicated rehabilitation. In addition, the restoration may be readily removed to allow evaluation of the health of the underlying tissues. Historically, many types of materials have been used. Wood, wax metals, vulcanite and many types of plastics have been used as rigid materials while flexible ones like gelatin glycerine mixtures, latex and elastic plastics have also found some usefulness. ${ }^{2}$

Presently, materials used for the maxillofacial prosthesis are vinyl plastics, polyurethane, silicone rubber and acrylic types.

\section{KEY WORDS}

Maxillofacial-Materials, HTV (High Temperature Vulcanized) Silicone, RTV Silicone.
Corresponding Author: Dr. Tanvi Rajesh Balwani, Near Ramakrishna Hotel, Subhedar Layout, Jhulelal Colony, Wardha, Maharashtra, India.

E-mail: tbalwani16@gmail.com

DOI: $10.14260 / j e m d s / 2020 / 729$

How to Cite This Article: Dubey SG, Balwani TR, Chandak AV, et al. Material in maxillofacial prosthodontics - a review. J Evolution Med Dent Sci 2020;9(44):3319-3324, $10.14260 /$ jemds/2020/729

Submission 25-06-2020,

Peer Review 22-09-2020

Acceptance 28-09-2020,

Published 02-11-2020.

Copyright (c) 2020 Surekha Godbole Dubey et al. This is an open access article distributed under Creative Commons Attribution License [Attribution 4.0 International (CC BY 4.0)] 


\section{BACKGROUND}

Maxillofacial prosthetics is defined as that branch of prosthodontics that is concerned with restoration and replacement of both of stomatognathic and associated facial structures by artificial substitutes that may or may not be removed. (GPT8) ${ }^{3}$ The facial structures which are affected by disease, injury, surgical or congenital defects can be treated or restored with the help of maxillofacial prosthesis. Although there are developments in the field of plastic surgery, still the need to rehabilitate small as well as large portions of the face with alloplastic materials is still needed. ${ }^{4}$

In the recent years, an intense growth in the demand for prosthetic rehabilitation of patients with facial defects is seen. Increasing awareness of cancer is resulting in earlier diagnosis and treatment. Unfortunately, many of the surgical techniques are extensive and thus leave large defects that compromise not only function and aesthetics, but also psychosocial status of the patient. These problems require prompt rehabilitation with surgery or prosthetics. However, surgical reconstruction is often not indicated when there is large defects or in highrisk patients. Prosthesis offers the advantage of quick, reversible and medically uncomplicated rehabilitation. In addition, the restoration may readily remove to allow evaluation of the health of underlying tissues. ${ }^{5}$

Presently, materials used for the maxillofacial prosthesis are vinyl plastics, polyurethane, silicone rubber and acrylic types. These new materials presented some excellent properties as well as major insufficiencies. A material has not been developed that resembles or duplicates human skin. ${ }^{6}$

The physical, biological and clinical properties of various maxillofacial materials have been studied by researchers to achieve clinical success and patient acceptance. The critical task to a facial prosthesis material is its clinical performance testing. Research should concentrate on two major goals.

a) Improving the physical and mechanical properties of the material, so that it will behave more like human tissue and increase the life of the prosthesis.

b) Finding colour stable agents or colouring facial prosthesis and develop a technique to simulate human skin colour. ${ }^{6}$

Properties of maxillofacial prosthetic materials are mentioned in Table 1, Table 2 and Table 3.

\begin{tabular}{|c|}
\hline High Tensile Strength \\
High Resistance to Abrasion \\
High Elongation \\
Low Coefficient of Friction \\
Low Glass Transition Temperature \\
Low Surface Tension \\
Low Thermal Conductivity \\
Low Specific Gravity \\
Odourless \\
Translucent \\
Pliable \\
\hline Table 1. Ideal Physical and Mechanical Properties \\
\hline
\end{tabular}

\begin{tabular}{|c|}
\hline Compatible with Supporting Tissues \\
\hline Nonallergic and Nontoxic \\
\hline Easy Cleanability without Loss of details at Margin or Surface \\
\hline Colour Stability \\
\hline Dimensionally Stable \\
\hline Flexibility Comparable to Tissue \\
\hline Inexpensive \\
\hline Resistance to Environment Discoloration \\
\hline Softness Maintained During Use \\
\hline Table 2. Ideal Biological Properties \\
\hline
\end{tabular}

\begin{tabular}{|c|}
\hline Adjustability \\
Chemically Inert after Processing \\
Dimensionally Stable \\
Ease of Processing \\
Long Shelf Life \\
Low Processing Temperature \\
Nontoxic Components \\
Nonporous after Processing \\
No Colour Change after Processing \\
Reusable Moulds \\
Short Processing Time \\
\hline Table 3. Ideal Processing Characteristics \\
\hline
\end{tabular}

PROSTHETIC RECONSTRUCTION MATERIALS

A number of materials are available for the fabrication of a successful prosthetic maxillofacial replacement.

\section{Impression Materials}

1. Reversible hydrocolloid

2. Irreversible hydrocolloid

3. Plaster of Paris

\section{Modelling Materials}

1. Modelling clay (sculptor's clay)

2. Plaster

3. Plastolene

4. Waxes

\section{Fabrication Materials}

1. Acrylic resins

2. Acrylic copolymers (Palamed, Polyderm)

3. Polyvinyl chloride and copolymers (Realistic, Mediplast, Prototype III)

4. Chlorinated polyethylene

5. Polyurethane elastomers (Epithane)

6. Latex

7. Silicone elastomers

7.1. HTV Silicones

7.1.1. Silastic 370, 372, 373, 4-4514, 4-4515,

7.1.2. PDM silicone.

7.2. RTV Silicones

7.2.1. Silastic 382,399

7.2.2. MDX 4-4210

7.2.3. Silastic 891

7.2.4. Cosmesil

7.3. A-2186

7.4. Foaming Silicones

7.4.1. Silastic 386.

7.4.2. Siphenylenes.

7.5. New Materials

7.5.1. Silicone block co-polymers.

7.5.2. Poly Phosphazones.

\section{Surgical Reconstruction Materials}

1. Alloplastic Implants.

2. Metals.

3. Tantalum.

4. Titanium. 
5. Stainless steel.

6. Autopolymerizing methyl methacrylate.

7. Heat polymerizing methyl methacrylate.

8. Polyethylene.

9. Silicone. $^{7}$

\section{Acrylic Resins}

Acrylic resins of methyl or polymethyl methacrylate most commonly used for denture base, became popular for facial prosthesis shortly after World War II. These materials because of their rigidity can be used successfully, in specific types of maxilla-facial defects. The material is readily available, and most dentists are familiar with physical and chemical properties and processing techniques. Both intrinsic and extrinsic colouration can be performed. Heat polymerizing methyl methacrylate is preferred over the autopolymerizing form because of the presence of free toxic tertiary amines in the latter. The colour stability when exposed to U.V light is better in polymerizing methyl methacrylate. ${ }^{8}$

\section{Acrylic Copolymers (Palamed, Polydrem)}

These are of acrylic and methacrylic acid. The esters in the monomer state are in liquid form converted into the polymeric form on processing. Palamed is available in laboratory pack and contains base powder, stain concentrates and solvent liquid for characterization of the finished prosthesis. These materials are available in three basic skin colour shades-pale, medium and dark. Palamed has a chemical bond to hard acrylic. This enables the sections of prosthesis to have supporting structures of hard acrylic. ${ }^{8}$

\section{Vinyl Plastisols (Realistic and Mediplast)}

The first vinyl resin, according to Clarke, was produced in 1833 but it was not until 1929, Ivan. Ostromislensky produced the plasticized polyvinyl chloride resin. The first PVC (PolyVinyl Chloride) produced specifically for prosthetic use was the lightly filled plasticized resins introduced by the Vrenon Ben shoff Co., 1943. This material can provide a very aesthetic, easy replacement of facial structure and presently is the most widely accepted material in the field of facial and stomatognathic prosthesis

\section{Advantages}

a. Flexible.

b. Acceptable initial appearance.

c. Adaptable to intrinsic and extrinsic colouration.

\section{Disadvantages}

a. Loss of plasticizer during the life of the prosthesis results in discolouration and hardening of prosthesis at the margins.

b. Poor dimensional stability.

c. Easily stain and degrade when exposed to U.V. light.

d. Requires metal moulds for curing at high temperature.

e. Lacks lifelike translucence and tends to absorb sebaceous cosmetics and solvents.

f. Short life expectancy of the prosthesis, 3 to 6 months.

g. Edges tear easily if thin and may require reinforcement with nylon fabric. ${ }^{8}$

\section{Chlorinated Polyethylene}

The polyethylene contains chlorine atoms and is compounded with low density calcium state and soybean oil.

Lewis and Castleberry reported testing of chlorinated polyethylene, a material which is similar to PVC in both chemical composition and physical properties.

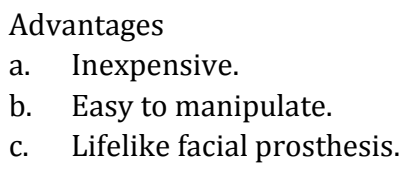

Disadvantages

a. Time consuming.

b. Colour instability.

c. Poor edge strength.

d. Short life span i.e., 3 - 4 months. ${ }^{8}$

\section{Polyurethane (Epithane 3)}

Polyurethane elastomers serve a variety of commercial and medical blood-contacting devices, cardiac replacement. On the other hand, Epithane-3 is currently in use for restoring facial defects. Epithane-3 was developed by Dan Rosa.

Advantages

a. The material has a life like feel on touch.

b. Excellent edge strength and elastic properties.

c. Extrinsically and intrinsically coloured to match skin tones.

d. Super cosmetic results can be obtained.

Disadvantages

a. Difficult to process.

b. Isocynates are moisture sensitive, stone molds are dehydrated before processing.

c. Poor colour stability

d. Short life expectancy, 3 - 6 months.

e. Poor compatibility with existing adhesive system. Free isocynates in cured restorations are potential for toxic reactions. ${ }^{8}$

\section{Silicone Elastomers}

It is chemically termed as polydimethyl silicone. Silicones were introduced around 1946, but only in the past few years, they have been used in the fabrication of maxillofacial prosthesis. Silicone elastomers were first used for external prosthesis by Barnhart in 1960, Silicones are currently the most popular of all of the facial prosthetic materials. Silicones consist of alternate chain silicone and oxygen atoms, which produce little or no inflammatory response in animals.

Silicones are classified into 3 groups according to their applications. The first classification is implant grade, which requires the material to undergo extensive testing and must meet or exceed FDA requirements. Recent health problems caused by silicone breast implant here created controversies regarding the safety of materials. The second classification is medical grade, which is approved for external use only. These are the materials most commonly used in fabrication of maxillofacial prosthesis. Adverse reactions caused by contact between medical grade silicone and human skin have not been 
reported. The third classification is industrial grade, which is mostly used for industrial applications. ${ }^{9}$

\section{HTV Silicones}

(Silastic 370, 372, 373 MDX 4-4514, MDX, 4-4515-4516)

Heat vulcanizing silicones are translucent, milky white, semisolid materials. The material may be supplied as one component or two component putty. The catalytic or vulcanizing agent of HTV silicones is Dichlorobenzoyl peroxide or platinum salt, depending upon the type of the polymerization used (condensation reaction or addition reaction). These silicones can be preformed into various shapes of alloplastic implantation for facial prosthesis.

\author{
Advantages \\ a. Excellent thermal stability. \\ b. Colour stable when exposed to U.V. light. \\ c. Superior strength. \\ d. Biologically inert.
}

Disadvantages

a. Low edge strength requires nylon reinforcement at the margins.

b. Opacity and life appearance.

c. Extrinsic coloration is difficult.

d. Requires milling device for incorporation of internal colorants.

\section{RTV (Room-Temperature-Vulcanizing) Silicones}

These are well known to dentists as an impression material designed for intra oral use. There are 2 major types of RTV silicones according to their curing mechanism - addition (e.g.: Silskin II, Silkskin) and condensation (e.g.: Cosmesil, Cosmesil $\mathrm{HC}_{2}$, Cosmesil HC4). Addition type silicones can be cured at elevated temperatures $\left(65^{\circ}-85^{\circ}\right)$ hence they are called low temperature-vulcanizing silicones. Carl Andres et al reported that majority of prosthodontics and prosthetists are using room temperature-vulcanized silicone products intrinsically coloured with dry pigments and artist's oils for the fabrication of extra oral prosthesis. ${ }^{10}$

\section{Advantages}

a. Ease of handling.

b. Speed and ease of processing.

c. Can be fabricated in an ordinary dental laboratory.

d. Excellent thermal stability and colour stable when exposed to U.V. Light.

e. Biologically inert.

\section{Disadvantages}

RTV silicones are weaker than vinyl plastisols. It exhibits zipper effects i.e., although the material can normally withstand considerable stress, a small nick or cut will allow it to literally fall apart.

a. High specific gravity, hence, the prosthesis might be heavy.

b. Stiff and less flexible.

c. Silicones also require adhesive materials used to hold the prosthesis to the skin.

d. Difficult to apply the adhesive and clean.

e. Poor wettability.
Silastic 382, 399 - The various silicone includes a filler, a stannous octate catalyst \& orthoalkyl silicate cross-linking agent. The polymerization is condensation reaction. Fillers, diatomaceous earth are used to improve strength. The properties of original RTV silicones (Silastic 382,399) are similar to HTV silicones.

MDX 4-4210 - This medical grade silicone elastomer has shown to be most popular among clinicians. From the results of the survey by Andreas, $41 \%$ of clinicians used this material for fabrication of maxillofacial prosthesis.

Silastic 891 - Udagama and Drane first reported the use of this material for fabrication of facial prosthesis it is also known as silastic medical adhesive silicone type A. It has gained popularity among clinicians. Twenty-five percent of clinicians reported the use of this material in a survey. It is a translucent, non-flowing paste, which polymerizes at room temperature on contact with moisture in the air. It can also be processed in a Gypsum mould. Metal moulds are not recommended because its surface may react with acetic acid, a by-product of polymerization. Advantages include no requirement of catalyst and compatibility with a wide range of colorants.

Cosmesil - Cosmesil was introduced in 1982 in the United Kingdom, this system contains silicone elastomer, on RTV sealant, colouring agents and accessories needed for preparing facial and body prosthesis. The Cosmesil system was eventually launched into the market in 2 versions of Condensation RTV silicones A (A basic one B) High compliance version, which was developed in 1993.

\section{Foaming Silicones}

Silastic 386 - A form of RTV silicone that has limited use in maxillofacial prosthesis is the foam-forming variety. The base silicone has an additive so that a gas is released when the catalyst, stannous octate is introduced. ${ }^{10-12}$

Siphenylenes - Siphenylenes are currently being developed and evaluated for use as a maxillofacial material according to Dr. Castleburry.: Siphenylenes are combinations of silicone and carbon polymers and therefore they have many of the advantages of both types of polymers. These materials exhibit many desirable properties including biocompatibility, resistance to degradation on exposure to U.V light and heat, improved edge strength and colorability.

\section{New Materials}

Silicone Block Copolymers - Silicone block co polymers are new materials under development to improve some of the weakness of silicone elastomers. It has been found that silicone block co polymers are more tear resistance than conventional cross-linked silicone polymers.

Polyphosphazines - Polyphosphazines flouroelastomers has been developed for use as a resilient denture liner and has the potential to be used as a maxillofacial prosthetic material. Modification of physical and mechanical properties of Polyphosphazines may be needed to satisfy the requirement for fabrication of maxillofacial prosthesis. ${ }^{11}$ 


\section{COLOURATION}

Accurate representation of skin color is essential in achieving aesthetics. Facial prosthesis remains the greatest challenge faced by the clinician. It requires a keen eye, good understanding of colour theory and application, meticulous attention to detail, practice and perseverance. Many approaches and techniques exist to achieve an accurate skin colour including trial and error mixing, shade guides, pigment dispersion systems, colorimeter or spectrophotometer..$^{13}$

Attempts have been made to record the amount of colorants used for custom formulation of base shade and extrinsic characterization for future needs. In past few years concerns with colour deterioration of maxillofacial prosthesis have directed most of the investigations on colour stability of base elastomer and colorants. Since the introduction of colour stable maxillofacial elastomers and colorants, there has been more emphasis on developing methods for colour matching of MFP to human skin. Now with advancing technology there have been developed computerized colour formulation which are described in Table 4.

Ma et al describes a technique for chair side verifications for facial prosthesis by making a processed specimen with different surface textures and thickness. ${ }^{14}$

The base colour can be selected by keeping the shade on the underside of the forearm, along the hairline, anterior to tragus and at the base of the helix. After identification of base colour, the mixing can be done by trial and error method. Adding small quantities of pigments to the silicone and comparing the mixture to the patient's skin tone. ${ }^{15}$

\begin{tabular}{|c|c|c|c|}
\hline Technique & 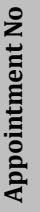 & Procedure & 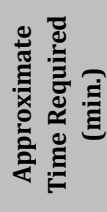 \\
\hline \multirow[t]{4}{*}{ Traditional } & 1 & Mix base colour & 90 \\
\hline & 1 & Mix laminar glazes & 90 \\
\hline & 2 & Reassess colour match, pack mould & 120 \\
\hline & 3 & Extrinsic coloration & 180 \\
\hline \multirow[t]{6}{*}{ Computerized } & 1 & Measure skin colour with spectrophotometer & 30 \\
\hline & 1 & Perform colour formulation & 120 \\
\hline & 1 & Evaluate colour formulated base colour & 30 \\
\hline & 2 & Evaluate colour formulated base colour & 90 \\
\hline & 3 & Mix laminar glazes & 120 \\
\hline & 4 & Extrinsic coloration & 180 \\
\hline \multicolumn{4}{|c|}{$\begin{array}{l}\text { Table 4. Timeline for Traditional vs. Computerized Colour } \\
\text { Formulation Colour Matching }\end{array}$} \\
\hline
\end{tabular}

Mark W. Beatty et al reported that addition of pigments (oil pigment) to the silicone may cause significant colour changes when exposed to U.V. radiation because of incompatibility between pigments and elastomer.

Stephen. O. Bertlett et al developed a modification of the technique for extrinsic coloration by using a silicone medical adhesive. The medical grade adhesive forms a tenacious bond to the base material, which will prevent the peeling of other layer of silicone rubber when cleaning and handling the prosthesis.

\section{RETENTION OF PROSTHESIS}

Retention of prosthesis is also a factor which plays a crucial role in providing retention, stability and support for the prosthesis. Retention to the maxillofacial prosthesis can be achieved by 4 ways - mechanical, adhesive, implants, and anatomic. Most facial prosthesis are retained with a medicalgrade adhesive. The commercially available adhesives are:

1. Daro adhesives (regular, extra strength and hydrobond).

2. Secure medical grade adhesive (B-400, 401 \& BT-402)

3. Secure extra strong adhesive (B-460, BT-460, B-520)

4. Double sided surgical tape biface polyethylene.

Selection of adhesives depends on patient tolerance, ease of application and removal, compatibility with the materials used for facial prosthesis.

Most cured silicones, because of low solubility and low surface energy, will not adhere to conventional tissue adhesives. The single component RTV silicones were developed to serve as adhesives for silicone prostheses (medical adhesive type A).

Skin-prep protective dressing, which is commonly used, protects the skin from trauma, abrasion, chaffing, and irritation by creating a physical waterproof barrier and allowing the skin to breathe. ${ }^{16,17}$

Wilbron et al found that the trauma was reduced when skin-prep protective dressing was used.

The effectiveness of adhesives depends on the bond strength between maxillofacial prosthesis material \& adhesives. Geetleman et al developed methods to measure the bond strength of various adhesives to maxillofacial materials against human skin.

The type of adhesives and the cleaning solution used for maxillofacial elastomer should be carefully chosen. It has been shown that adhesives and solvents can have adverse effects on physical and optical properties or maxillofacial elastomers. ${ }^{18,19}$

Kiat Amnuay et al reported that skin-prep protective dressing improved the retentive properties of adhesives. It was found that a secure medical adhesive was much more retentive than epithane- 3 adhesive.

The bond strengths of silicone rubber adhesives to the skin decreased during, the course of the day because of perspiration and normal body motion. ${ }^{20}$

Kiat Amnuay et al investigated the effect of application of second layer of adhesive on retention of silicone adhesives. He found that the bond strength of silicone elastomer to skin decreased over on 8-hour interval. Reapplication of skin medical adhesives over the existing adhesive improves the bond strength.

The removal of adhesives from the skin is also problematic. So, solvents such as Uni-solvent adhesive remover are often used.

Reports on evaluation of clinical performance and biocompatibility of medical adhesives are limited. Udagama reported that adhesives and the prosthetic material applied together caused more irritation than the adhesive alone.18 Dahl et al investigated the irritative potential of various prosthetic adhesives by using an invitro technique. He found that irritation may be due to the adhesives and products containing ethyl acetate especially if the skin is thin and vulnerable. ${ }^{18}$ 
An alternative to medical skin adhesives is the use of osseointegrated implants to retain the prosthesis. Incorporation of retentive elements (bar-clips or magnets) within the prosthesis reduces the overall bulk of the maxillofacial prosthetic material thereby reducing the strength of the prosthesis.

Since some patients are poor candidates for implant intervention (compromised tissue beds, financial limitations), they have to rely on skin adhesives to retain their facial prosthesis.

The use of tissue undercuts is an alternative method of mechanical retention of facial prosthesis. The soft, flexible nature of silicone material appears to use tissue undercuts for retention. However, the abrasive nature of the natural prevents its use for retention.

Udagama et al found that mechanically retained facial prosthesis depends on the nature of material, physical condition and history of supporting tissues.

\section{CONCLUSIONS}

Maxillofacial prosthesis is replacement of the missing facial parts by artificial substitutes. Anciently prosthetic restoration of facial defects was limited due to unavailability of adequate materials. Historically, many types of materials have been used such as wood, wax metals, vulcanite and many types of plastics have been used as rigid materials while flexible ones like gelatin glycerine mixtures, latex and elastic plastics were also used.12

Recent materials used for the maxillofacial prosthesis are vinyl plastics, polyurethane, silicone rubber and acrylic types. These new materials have exhibited some excellent properties, but each material has its own disadvantages. ${ }^{20} \mathrm{~A}$ material has not yet emerged that completely resembles or duplicates human skin.

Financial or other competing interests: None.

Disclosure forms provided by the authors are available with the full text of this article at jemds.com.

\section{REFERENCES}

[1] Lewis DH, Castleberry DJ, Fischer TH. New and improved elastomes for extraoral maxillofacial prosthesis. J Dent Research 1977;51:23-8.

[2] Kiat-Amnuay S, Gettleman L, Goldsmith JL. Effect of multiadhesive layering on retention of extraoral maxillofacial silicone prostheses in vivo. J Prosthet Dent 2004;92(3):294-8.

[3] The glossary of prosthodontic terms. J Prosthet Dent 2005;94(1):10-92.

[4] Kiat-Amnuay S, Gettleman L, Khan Z, et al. Effect of adhesive retention of maxillofacial prosthesis. Part 2: Time and reapplication effects. J Prosthet Dent 2001;85(5):438-41.
[5] Abdelnnabi MM, Moore DJ, Sakumura JS. In vitro comparison study of MDX-4-4210 and polydimethyl siloxane silicone materials. J Prosthet Dent 1984;51(4):523-6.

[6] Andres CJ, Haug SP, Munoz CA, et al. Effects of environmental factors on maxillofacial elastomers: Part I- Literature review. J Prosthet Dent 1992;68(2):327-30.

[7] Maller US, Karthik KS, Maller SV. Maxillofacial prosthetic materials- past and present trends. J Indian Acad Dent Spec 2010;1(2):25-30.

[8] Haug SP, Moore K, Andres CJ. Color stability and colorant effect on maxillofacial elastomers. Part II: Weathering effect on physical properties. J Prosthet Dent 1999;81(4):423-30.

[9] Lewis DH, CastleberryDJ, Fischer TH. New and improved elastomes for extraoral maxillofacial prosthesis. J Dent Research 1977;51:23-8.

[10] Nimonkar S, Belkhode VM, Sathe S, et al. Prosthetic rehabilitation for hemimaxillectomy. Journal of Datta Meghe Institute of Medical Sciences University 2019;14(2):99-102. https://doi.org/10.4103/jdmimsu.jdmimsu_40_19.

[11] Borle RM, Nimonkar PV, Rajan R. Extended nasolabial flaps in the management of oral submucous fibrosis. British Journal of Oral \& Maxillofacial Surgery 2009;47(5):382-5. https://doi.org/10.1016/j.bjoms.2008.08.019.

[12] Choudhary AB, Motwani MB, Degwakar SS, et al. Utility of digital volume tomography in maxillofacial trauma. Journal of Oral and Maxillofacial Surgery 2011;69(6):e135-e40. https://doi.org/10.1016/j.joms.2010.07.081.

[13] Hatamleh MM, Watts DC. Bonding of maxillofacial silicone elastomers to an acrylic substrate. Dental Materials 2010;26(4):387-95.

[14] Ma T, Hicken SC, Buchanan CR, et al. Chairside color verification for facial prosthesis. J Prosthet Dent 1988;60(2):219-21.

[15] Chalian VA, Drane JB, Standish MS. Maxillofacial prosthetics- multidisciplinary practice. Williams \& Wilkins Company, 1972.

[16] Kiat-amnuay S, Mekayarajjananonth T, Powers JM, et al. Interactions of pigments and opacifiers on color stability of MDX4-4210/type A maxillofacial elastomers subjected to artificial aging. J Prosthet Dent 2006;95(3):249-57.

[17] Kiat- Amnuay S, Beerbower M, Powers JM, et al. Influence of pigments and opacifiers on color stability of silicone maxillofacial elastomer. Journal of Dentistry 2009;37(1):e45-e50.

[18] Dahl JE, Polyzois GL. Irritation test of tissue adhesives for facial prostheses. J Prosthet Dent 2000;84(4):453-7.

[19] Polyzois GL. Color stability of facial silicone prosthetic polymers after outdoor weathering. J Prosthet Dent 1999;82(4):447-50.

[20] Han Y, Powers JM, Kiat-Amnuay S. Effect of opacifiers and UV absorbers on pigmented maxillofacial silicone elastomer, part 1: color stability after artificial aging. J Prosthet Dent 2013;109(6):397-401. 